\section{THU0646 INTERCHANGEABILITY FROM INFLIXIMAB ORIGINATOR TO INFLIXIMAB BIOSIMILAR: EFFICACY AND SAFETY IN A PROSPECTIVE OBSERVATIONAL STUDY ON 89 PATIENTS}

Y. Presberg ${ }^{1}$, V. Foltz ${ }^{2}$, C. L'Amour ${ }^{2}$, P. Tilleul ${ }^{1}$, D. Zerkak ${ }^{2}$, C. Gard ${ }^{1}$, B. Fautrel ${ }^{2,3}$. ${ }^{1}$ Pharmacy; ${ }^{2}$ Rheumatology, AP-HP pitié salpetrière hospital; ${ }^{3}$ Université Pierre et Marie Curie (UPMC)-Paris 6, Paris, France

Background: Biosimilar biologic disease modifying anti-rheumatic drug (bsDMARD) are supposed to offer a real economic advantage for these diseases for the same health benefit. While the administration of a biosimilar to a new patient is widely accepted, the switch from a biologic originator DMARD (boDMARD) to its biosimilar remains questioned.

Objectives: This study aimed to assess the safety and efficacy of switching from an IV anti-TNF boDMARD to its bsDMARD in patients with rheumatic arthritis, spondyloarthritis, psoriatic arthritis, juvenile arthrtitis and uveitis.

Methods: Prospective real-life study in the rheumatology unit of La PitiéSalpêtrière Hospital, from February 2016 to December 2016. Patients initially treated with the boDMARD infliximab (RemicadeO), all switched to infliximab bsDMARD (InflectraO) after patient's information by a rheumatology nurse. The dose and administration schedule remained unchanged at the switch. Our primary endpoint was infliximab maintenance rate. Secondary endpoints were evolution of disease activity, as well as evolution of both infliximab trough levels and anti-drug antibody (ADA) serum levels before and after the switch. In addition, safety events were also monitored.

Results: We identified 89 patients - spondyloarthritis $(61 \%)$, rheumatic arthritis $(27 \%)$, psoriatic arthritis (11\%), juvenile arthritis and uveitis (1\%) - in rheumatology who have been switched to boDMARD infliximab to its bsDMARD. Clinical assessment was based on DAS28 (peripheral joint involvement) or BASDAI (axial involvement) scores. 93\% of patients remained treated with infliximab: 6 patients (7\%) stopped the treatment due to a loss of efficacy. As shown in Figure 1, there is no significant change in disease activity before and after having switched patients to infliximab biosimilar.The ADAdosage were detected in 10 patients, 7 with high titer $(>200 \mathrm{ng} / \mathrm{mL}$ ) and 3 with moderate titer (between 50 and 200 $\mathrm{ng} / \mathrm{mL})$. It was significantly correlated with low trough levels of IFX $(<0,3 \mu \mathrm{g} / \mathrm{mL})$. Among them, 6 (4 with high titer; 2 with moderate titer) also had a poor clinical response before switch (refractory patients). 4 ADA-positive patients ( 3 with high titer; 1 with moderate titer) were still good clinical responders.

Conclusions: The interchangeability of infliximab boDMARD to its bsDMARD does not rise any efficacy and safety concern in our experience. The role of drug and ADA monitoring during the switch remains to be further explored.

[1] Jørgensen K et al. (2016) LB15 - Biosimilar infliximab (CT-P13) is not inferior to originator infliximab: results from the 52-week randomized NOR-SWITCH trial. BioDrugs 2017 Jan 24. doi: 10.1007/s40259-017-0210-0,

[2] Interchangeability of Biosimilars: A European Perspective, Kurki P, van Aerts L, Wolff-Holz E, Giezen T, Skibeli V, Weise M.

Disclosure of Interest: None declared

DOI: 10.1136/annrheumdis-2017-eular.6586

\section{THU0647 INTERACTIONS BETWEEN STEPS PER DAY AND RISK FACTORS FOR OSTEOARTHRITIS ON MRI-DETECTED OSTEOPHYTES IN A POPULATION BASED COHORT STUDY}

Z. Zhu ${ }^{1,2}$, W. $\operatorname{Han}^{1}$, S. Zheng ${ }^{1}$, K. Wang ${ }^{1}$, T. Winzenberg ${ }^{1}$, F. Cicuttini ${ }^{3}$ C. Ding ${ }^{1}$, G. Jones ${ }^{1}$ on behalf of Musculoskeletal Uni, Menzies Institute for Medical Research. ${ }^{1}$ Musculoskeletal Unit; ${ }^{2} 12779$, Hobart: ${ }^{3}$ Department of epidemiology and Preventive Medicine, Monash University, Melbourne, Australia

Background: It is clear that exercise helps the symptoms of osteoarthritis. However, the relationship between physical activity (PA) and progression of knee osteoarthritis $(\mathrm{OA})$ remains controversial. Moreover, no guideline on the amount of PA to prevent OA progression in general older population has been recommended, and it is still uncertain whether PA should be promoted among subgroups with knee OA such as female gender, obesity, radiographic osteoarthritis (ROA) and knee injury history. Osteophyte (OP) has long been viewed as a defining structural feature of knee OA. Recently, MRI-detected OPs have been shown to be more sensitive to change than radiographs.

Objectives: The aims of this study were to describe the longitudinal association between objectively-measured PA and knee MRI-detected (OPs), and to test the interactions between PA and gender, obesity, ROA or knee injury history on the increases in MRI-detected OPs.

Methods: 408 community-dwelling adults aged 51-81 were randomly selected from local community and measured at baseline and 2.6 years later. T1-weighted fat suppressed MRI was used to evaluate knee OPs at baseline and after 2.6 years. PA was assessed at baseline by pedometers (steps per day) and categorized as 3 groups: low PA ( $\leq 7499$ steps per day), moderate PA (7500 to 9999 steps per day) and high PA ( $\geq 10000$ steps per day). Radiographs were obtained and scored for individual features of radiographic osteoarthritis (ROA). Knee injury history was recorded by questionnaire and body mass index (BMI) was calculated. Logistic regression and log binomial regression were used in longitudinal analyses.

Results: In total study sample, doing moderate PA was associated with reduced risk of an increase in MRI-detected OPs, comparing to low PA (RR=0.73, $p=0.03$ ).
There were significant interactions between PA and gender, obesity, ROA or knee injury history (all $p<0.05)$ for an increase in MRI-detected OPs. In stratified analyses, moderate PA was protective against an increase in MRI-detected OPs in females $(\mathrm{OR}=0.23, \mathrm{p}<0.01)$, obese participants $(\mathrm{OR}=0.23, \mathrm{p}<0.01)$, participants with $\mathrm{ROA}(\mathrm{OR}=0.45, \mathrm{p}=0.02)$ and participants with knee injury history $(\mathrm{OR}=0.05$, $\mathrm{p}=0.02$ ). These significant associations still existed after further adjustments for age, sex, BMI, ROA and/or knee injury history (where appropriate). High PA was not associated with an increase in MRI-detected OPs in total sample or the stratified analyses but there were relatively few in this category.

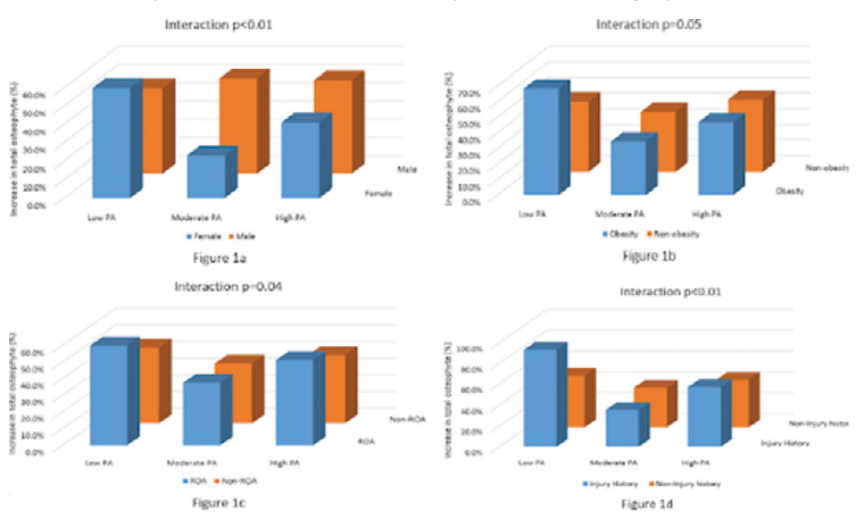

Conclusions: Moderate PA is protective for change in MRI-detected OPs in older adults, especially in those with female gender, ROA, obesity and knee injury history. This implies older people with above risks of knee OA may need to aim for a range from 7500 to 9999 steps per day.

References:

[1] Dore DA, Winzenberg TM, Ding C, Otahal P, Pelletier JP, Martel-Pelletier J, et al. The association between objectively measured physical activity and knee structural change using MRI. Ann Rheum Dis 2013; 72: 1170-1175.

Acknowledgements: The authors thank the participants who made this study possible, and acknowledge the role of the staff and volunteers in collecting the data, particularly research nurses Boon C and Boon P. Warren R assessed MRIs and $\mathrm{Dr}$ Srikanth $\mathrm{V}$ and $\mathrm{Dr}$ Cooley $\mathrm{H}$ assessed radiographs.

Disclosure of Interest: None declared

DOI: 10.1136/annrheumdis-2017-eular.1162

\section{THU0648 USE OF OUTPATIENT RHEUMATOLOGIC HEALTH CARE SERVICES BEFORE AND AFTER SWITCH FROM ORIGINATOR TO BIOSIMILAR INFLIXIMAB}

B. Glintborg ${ }^{1}$, J. Sørensen ${ }^{2,3}$, M.L. Hetland ${ }^{1} .{ }^{1}$ The DANBIO registry and Copenhagen Center for Arthritis Research (COPECARE), Center for

Rheumatology and Spine Diseases, Centre of Head and Orthopaedics, Glostrup; ${ }^{2}$ University of Southern Denmark, Odense, Denmark; ${ }^{3}$ Royal College of

Surgeons in Ireland, Dublin, Ireland

Background: According to Danish national guidelines issued in 2015, pts (pts) with inflammatory rheumatic diseases in routine care treatment with originator infliximab (INX) must switch to the cheaper biosimilar infliximab (CT-P13). This switch was done for economic reasons (CT-P13 64\% cheaper than INX). Since any treatment change potentially induces additional guidance of pts and use of hospital services, it is relevant to investigate whether the switch affected use of hospital resources.

Objectives: To study difference in rates of outpatient activities and services provided 6 months before vs after the switch to CT-P13 for pts treated at rheumatologic departments which provide infliximab treatment.

Methods: The switch population and switch dates were identified in the Danish quality registry, DANBIO (1). Vital status was validated in the Central Person Registry. The Danish National Patient Registry (NPR) provided information regarding outpatient contacts and services. The use of 16 types of services relevant to the rheumatologic specialty was identified and included outpatient visits, infliximab infusion, nurse counselling, phone consultation, and rheumatologic ultrasound. Number of days with these services was counted. Services performed on the switch date were analyzed separately. Thus, for each patient the following rates were calculated 6 months before and after the switch: 1) days with at least one outpatient service provided, and 2) number of services provided. In addition, the weekly rate of days with services was calculated.

Only pts who were alive and could be followed in the NPR for 6 full months after the switch were included.

Paired t-tests were used to compare rates before and after switch. A graphical interrupted time series analysis was conducted for weekly number of services provided.

Results: Among 802 pts identified, 769 had available NPR outpatient data from hospital departments of rheumatology. The 769 pts had 1484 outpatient treatment contacts at the included rheumatology departments with a total of 9243 days with services provided (including 693 on the switch date). The mean rate of days with services provided was 5.4 before the switch and 5.7 after switch $(p=0.0003)$. The 